\title{
Arachnoiditis, CTCAE
}

National Cancer Institute

\section{Source}

National Cancer Institute. Arachnoiditis, CT CAE. NCI Thesaurus. Code C143295.

A disorder characterized by inflammation of the arachnoid membrane and adjacent subarachnoid space. 